\title{
The Conundrum of Dual Primary Malignancies: Four Years' Experience of a Single Tertiary Care Institute in India
}

\begin{abstract}
Background: Encountering more than one malignancy in a cancer patient is no longer uncommon; this increasing incidence is mostly attributable to the improvements in life expectancy, awareness, and diagnostic facilities. This article aims to highlight this institute's experience in diagnosis and treatment of patients of multiple primary malignancies and a comprehensive review of literature. Materials and Methods: This is a descriptive study of retrospectively collected data of a single institution over 4 years from 2013 to 2016 . Known cases of cancer who were diagnosed with a second primary malignancy were included in the study. Various details such as age, sex, site of disease, temporal relation of two cancers (synchronous or metachronous), family history, tobacco use, treatment given, and survival at 1 year were recorded, organized in a tabular form, analyzed, and described. Results: A total of 29 cases of dual malignancies comprising $0.74 \%$ of a total of 3879 patients of cancer were encountered. Seventy-two percent of the cases were metachronous and 5 years was the mean time interval between tumors. There was a female preponderance, and the average age was 56 years. Breast was the most common site of malignancy. At 1 year from diagnosis of second primary, $69 \%$ of the patients were alive and $27 \%$ were disease-free. Conclusion: Second primary in a patient of cancer is becoming increasingly common and the suspicion of the same should always be borne in mind during follow-up. Prognosis as well as intent of treatment depends on respective stages of the two malignancies.
\end{abstract}

Keywords: Dual malignancy, metachronous, synchronous

\section{Introduction}

Multiple primary malignancy (MPM) in a cancer patient is not a new or a very rare occurrence. The diagnosis of a second or a third primary is very often not easy to arrive at due to the possibility of recurrent or secondary lesions from the first malignancy confounding the issue. This can lead to delay in initiation of appropriate treatment and can affect overall prognosis and survival. The most common presentation of MPMs is as dual malignancies. ${ }^{[1,2]}$

The concept of MPMs in one individual was first described by Billroth in $1889 .{ }^{[3]}$ In 1921, Owen published a report highlighting the possible causes of MPMs wherein they found $4.7 \%$ of cases of multiple growths in 3000 cases of malignancy. ${ }^{[4]}$ Warren and Gates $^{[5]}$ published the first literature about multiple cancers in 1932 and described the following salient points for their diagnoses: (a) each of the tumors should be malignant with proven histology, (b) they should be histologically distinct from each

This is an open access journal, and articles are distributed under the terms of the Creative Commons Attribution-NonCommercial-ShareAlike 4.0 License, which allows others to remix, tweak, and build upon the work non-commercially, as long as appropriate credit is given and the new creations are licensed under the identical terms.

For reprints contact: reprints@medknow.com other, and (c) the exclusion of metastasis should always be made. In 1977, Moertel[5,6] further refined the concept and classified multiple primary cancers and multicenter cancers into various groups based on their tissue and organ of origin [Table 1].

MPMs can be divided into synchronous or metachronous on the basis of the time interval between the diagnosis of the two primaries. As per Moertel et al., ${ }^{[7]}$ synchronous or "simultaneous" malignancies are those primary tumors which occur in the same patient within 6 months of each other, whereas metachronous or "interval" malignancies are those that occur in the same patient separated by a period that is $>6$ months. Although this definition of synchronous and metachronous tumors is the one most commonly used, it is not the only one and other researchers have used 12 months and other varying time intervals to define the temporal relation between MPMs.

Indian data on MPMs are limited to a few case reports or case series, ${ }^{[8-10]}$ with limited or no follow-up. Collection of long-term

How to cite this article: Bisht N, Singh S, Sarin A,
Gupta S, Singh HP, Kapoor A, et al. The conundrum
of dual primary malignancies: Four years' experience
of a single tertiary care institute in India. Indian J Med
Paediatr Oncol 2019;40:521-30.

How to cite this article: Bisht N, Singh S, Sarin A, Gupta S, Singh HP, Kapoor A, et al. The conundrum of a single tertiary care institute in India. Indian J Med Paediatr Oncol 2019;40:521-30.

\author{
Niharika Bisht ${ }^{1}$, \\ Sankalp Singh', \\ Arti Sarin ${ }^{1}$, \\ Samir Gupta ${ }^{1}$, \\ Harinder Pal Singh ${ }^{1}$, \\ Amul Kapoor ${ }^{1}$, \\ Deepak Mulajker ${ }^{1}$, \\ Richa Joshi', \\ Abhishek \\ Purkayastha', \\ Prabha Shankar \\ Mishra ${ }^{1}$, \\ Divya Shelly²
}

${ }^{1}$ Malignant Disease Treatment Centre, Command Hospital (SC), ${ }^{2}$ Department of Pathology, Armed Forces Medical College, Pune, Maharashtra, India

Submitted: 25-Mar-2018 Accepted in Revised Form: 21-Jun-2018

Published: 17-Feb-2020

Address for correspondence: Dr. Sankalp Singh, Malignant Disease Treatment Centre, Command Hospital (SC), Pune - 411 040, Maharashtra, India.

E-mail: sankalpsingh9@gmail. com

Access this article online

Website: www.ijmpo.org

DOI: 10.4103/ijmpo.ijmpo_69_18 Quick Response Code:

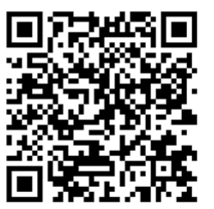




\begin{tabular}{ll}
\hline $\begin{array}{c}\text { Table 1: Classification of multiple primary malignant } \\
\text { neoplasms }\end{array}$ \\
\hline Category Description \\
\hline I & $\begin{array}{l}\text { Multiple primary malignant neoplasms of multicenter } \\
\text { origin }\end{array}$ \\
A. The same tissue and organ \\
B. A common, contiguous tissue shared by different \\
organs \\
C. The same tissue in bilaterally paired organs \\
II $\quad \begin{array}{l}\text { Multiple primary malignant neoplasms of different } \\
\text { tissues or organs }\end{array}$ \\
III $\quad \begin{array}{l}\text { Multiple primary neoplasms of multicenter origin plus a } \\
\text { lesion (s) of different tissue or organ }\end{array}$ \\
\hline
\end{tabular}

data with adequate follow-up and survival analysis is crucial to understanding the natural history of patients with MPMs in our country. In this retrospective analysis, we aim to report the incidence of dual malignancy seen in our practice and the demographic distribution, the patient and disease characteristics, and the management offered in such cases.

\section{Materials and Methods}

This is a descriptive study of retrospectively collected data from the cancer registry of a tertiary cancer hospital. Records of patients registered and treated at our center over a period of 4 years from January 2013 to December 2016 were perused. All patients who were found to have histologically proven MPMs were included in the study. Localized or disseminated recurrence of the same histological tumor was not included in the study.

Various demographic details such as patient's age at the time of each tumor diagnoses, sex, any relevant family history and history of tobacco usage were recorded. Similarly, disease details such as site of tumor, stage at presentation, histology, and time interval between the two diagnoses were also noted. If the two primary malignancies were diagnosed within 6 months of each other, they were labeled as synchronous, and if the time interval between their diagnoses was $>6$ months, the second neoplasm was categorized as a metachronous tumor. Among synchronous tumors, the one that was diagnosed earlier was deemed to be the first primary and the one detected subsequently was classified as the second primary.

Complete treatment details including surgery, radiotherapy, and systemic therapy were also registered. Only patients with a recorded follow-up period of 1 year from the diagnosis of second primary were included in the study. Patients who did not follow up in the hospital were included only if they or their caregiver could be contacted via telephone and disease status at 1 year from diagnosis could be recorded. The patients were divided into four groups as given below:

1. Alive and disease free - Patient alive at 1 year with no evidence of residual disease
2. Alive with stable disease - Patient alive at 1 year with residual disease that has either decreased partially or not increased in spread or volume since diagnosis

3. Alive with progressive disease - Patient alive at 1 year with residual disease that has increased in spread or volume since diagnosis

4. Dead - Patient who has died within 1 year of diagnosis.

Survival of patients with metachronous and synchronous tumors at 1 year was depicted using Kaplan-Meier graphs, and the survival probability between the two was compared using the log-rank test. Similar graphs were also generated to show the survival of different stages of the second primary cancer. All the collected data were organized in a tabular form, and the patterns of demographic, disease, treatment, and survival data were analyzed and reported.

\section{Results}

The list of patients included in the study is given in Table 2. A total of 29 cases of dual malignancies were found and included in our study comprising $0.74 \%$ of a total of 3879 cancer patients seen over a period of 4 years. There was a clear female predominance with $65.5 \%$ (19) of the patients being women. Interestingly, this female predominance was limited to the age group below 60 years, while the male sex was much more common in the age group above 60 years [Figure 1].

The mean age at presentation of first malignancy was 54 years, while the age at presentation of second malignancy ranged from 29 to 79 years with the average being 56 years. There were only two patients out of the 29 patients who had a history of a first- or second-degree relative suffering from cancer.

Ten out of the $29(35 \%)$ patients gave a history of using tobacco in any form. Six of these 10 patients had both such primaries that have tobacco use as a direct etiological factor (head and neck, lung, and esophagus cancers).

Breast was the most common site of cancer in the study with nine cases $(16 \%)$ closely followed by head and neck (oral cavity, oropharynx, larynx, and hypopharynx) with 7 (12\%) and lung with $6(10 \%)$ cases, respectively [Figure 2].

Carcinoma breast was also the most common first primary with seven such cases, while among the second primaries, lung carcinoma was the most common with five patients diagnosed with it.

All cancers were staged as per the $7^{\text {th }}$ Edition of AJCC staging system (2010). Among the first primaries, five out of the $29(17 \%)$ patients were in Stage IV at presentation, whereas among the second primaries, 16 (55\%) patients were in Stage IV at presentation. Eight $(28 \%)$ patients had synchronous cancers while $21(72 \%)$ had metachronous cancers. Among those with metachronous tumors, the time interval between the two tumors ranged between 23 years and 1 year, with the mean time interval being 5.33 years. 


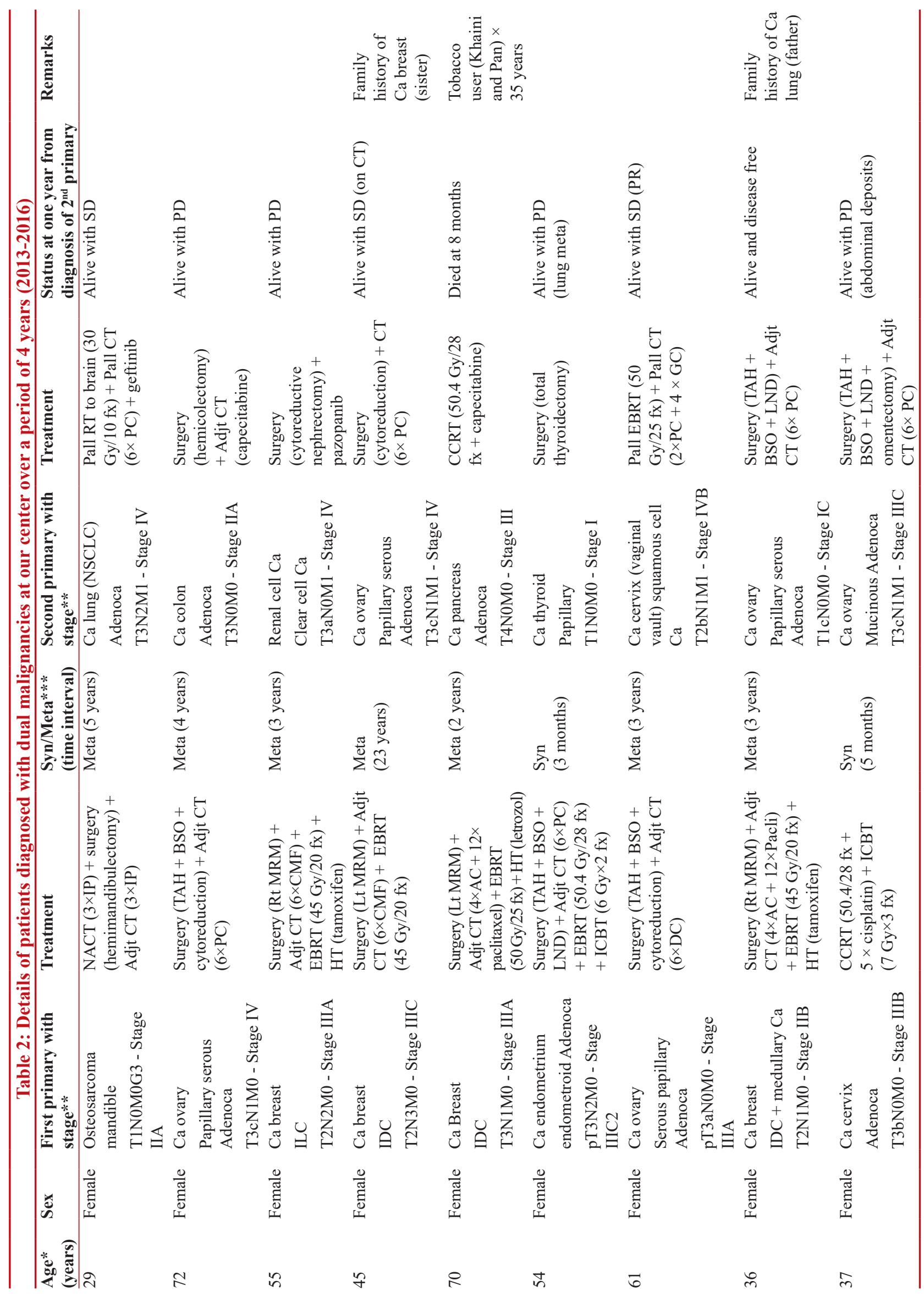




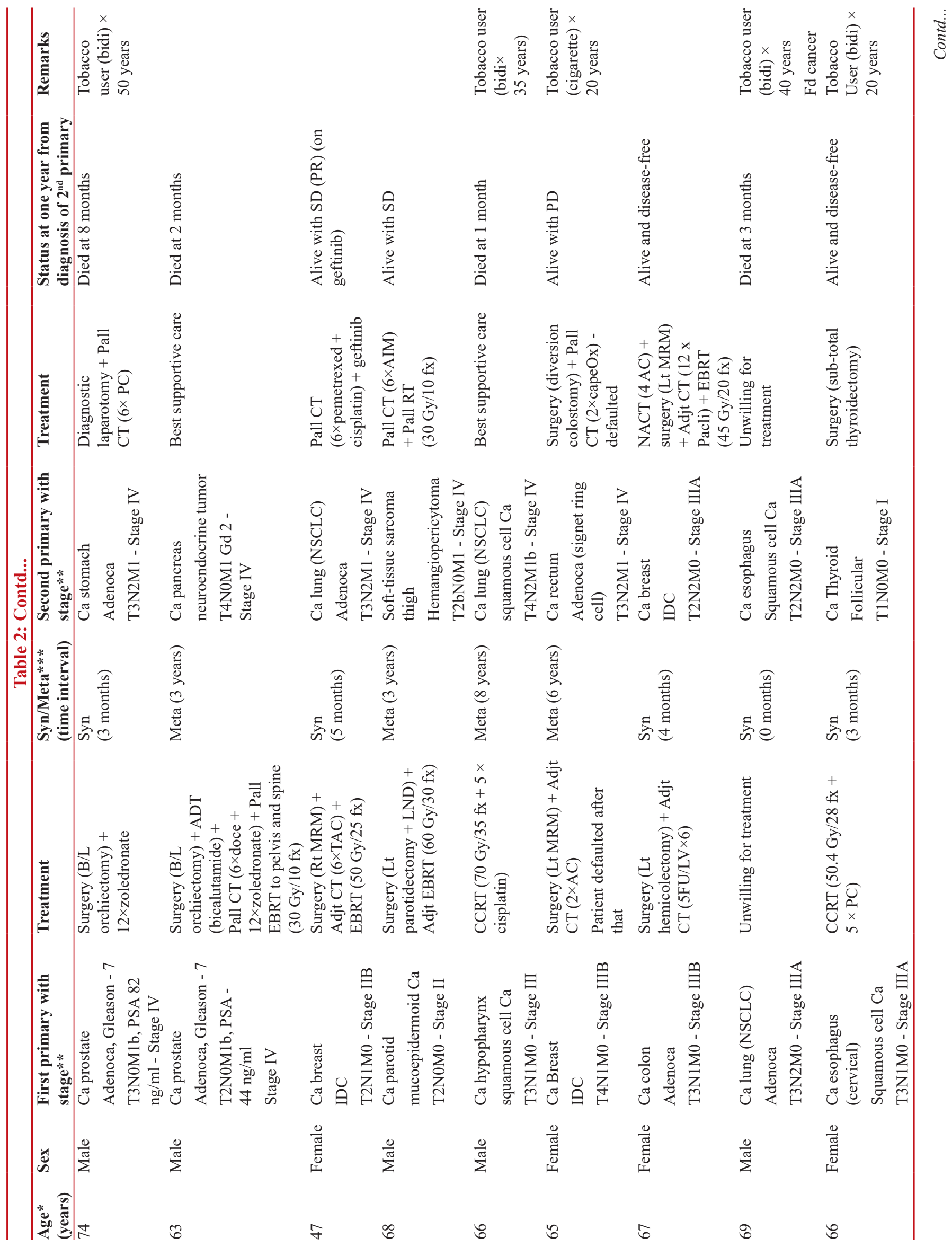




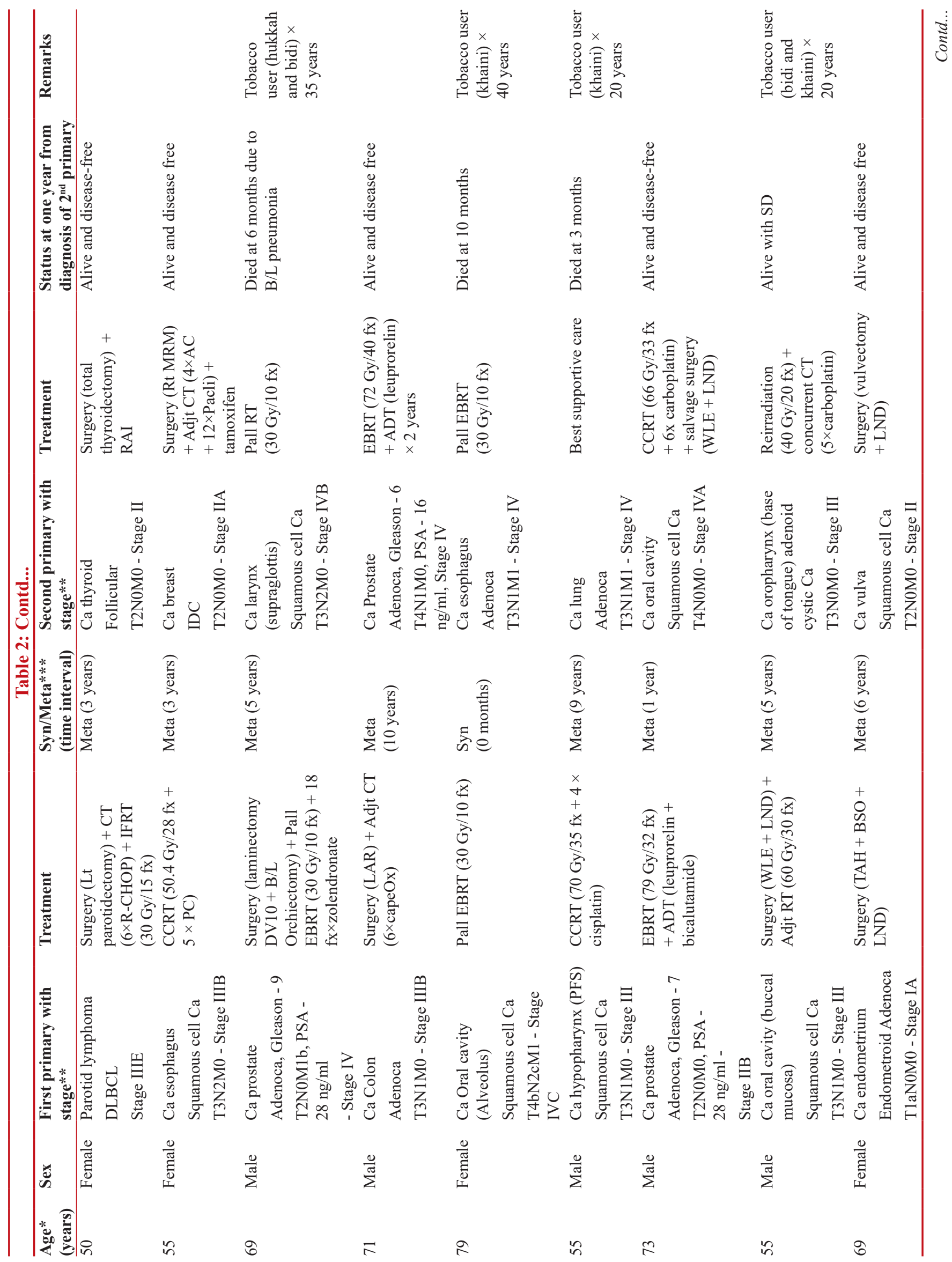



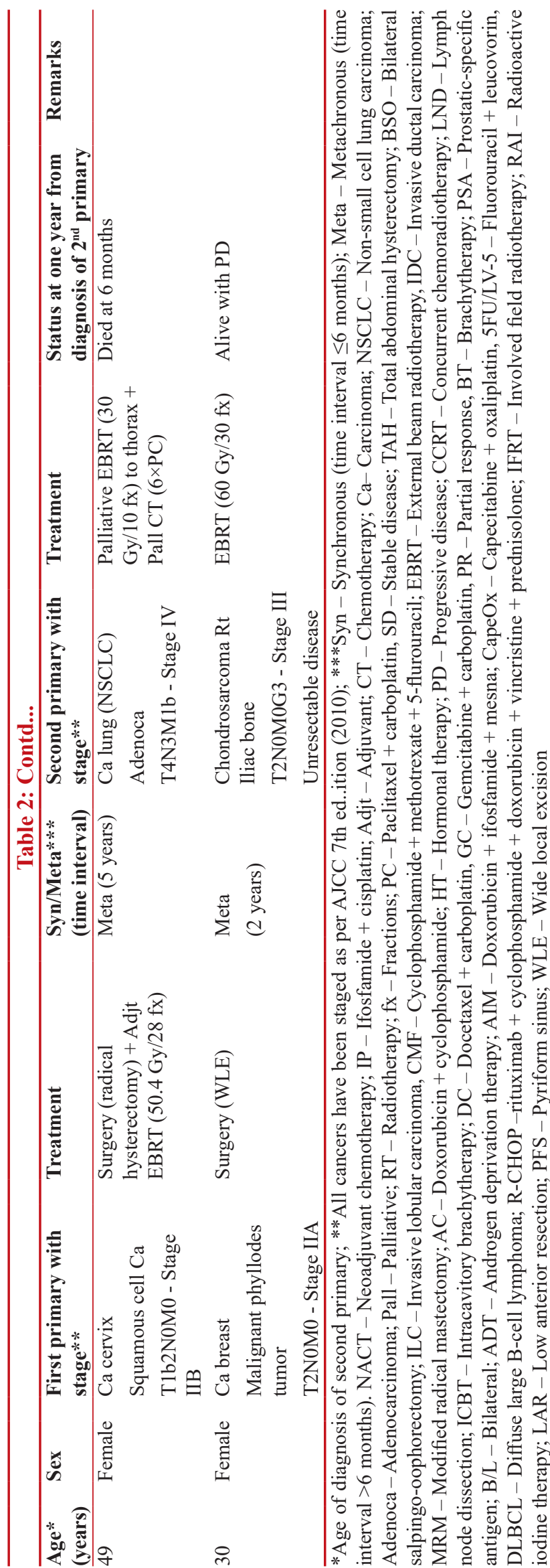

At the presentation of first primary, locoregional therapies in the form of surgery or radiotherapy were used in 21 and 19 cases, respectively, while at the presentation of second primary, the number of cases treated with surgery and radiotherapy reduced to 14 and 13, respectively. Systemic therapy in the form of chemotherapy, hormonal therapy, and targeted therapy was used in 22 and 18 patients in the treatment of first and second primary, respectively. There were also three patients who were offered only supportive care at presentation of second primary and one patient who refused treatment for both first and second primary cancers [Figure 3].

The intent of treatment was curative in 24 cases at presentation of first primary and palliative in $4(14 \%)$ cases, whereas at presentation of second primary, the intent was curative in 16 and palliative in 12 (41\%) cases. One patient refused treatment as mentioned earlier.

Follow-up for all patients was recorded up to 1 year from the diagnosis of second primary malignancy. Status of the patients at 1 year is displayed as a pie-chart distribution[Figure 4].

The proportion of deaths in the metachronous (7 out of 21 or $33.33 \%$ ) and synchronous (3 out of 8 or $37.5 \%$ ) groups were similar, and no statistical difference in survival probability was seen $(P=0.9201)$ [Figure 5]. The KaplanMeier graph showing survival as a function of stage of the second primary shows that all deaths occurred in Stages III and IV only [Figure 6].

\section{Discussion}

Over the past few decades, there appears to be a sharp upward trend in the occurrence of MPMs with the prevalence ranging from $0.7 \%$ to $11.7 \%$ among various populations. ${ }^{[11]}$ The possible reasons for this can be manifold including the improved survival and life expectancy of cancer patients due to improved treatment modalities, availability of better diagnostic technologies, and more stringent surveillance of cancer survivors. ${ }^{[12-16]}$ In our study, $0.74 \%$ of cancer patients developed a second malignancy over a period of 4 years. This is at the lower limit of the range and is consistent with the lower incidence of cancer in India compared to the Western countries. Another contributing factor may have been the fact that we have only considered solid organ malignancies in our study and not hematological ones.

An individual with previous history of cancer has a $14 \%$ higher risk of developing subsequent cancer than would be expected in the general population. ${ }^{[17]}$ This increased incidence could be because of possible genetic susceptibility as well as exposure to environmental carcinogens such as tobacco, alcohol, viruses, and certain chemicals. The treatment of primary malignancy by chemotherapy and radiotherapy may also contribute to this as both ionizing radiation and cytotoxic agents (etoposide, cyclophosphamide, etc.,) can cause DNA 


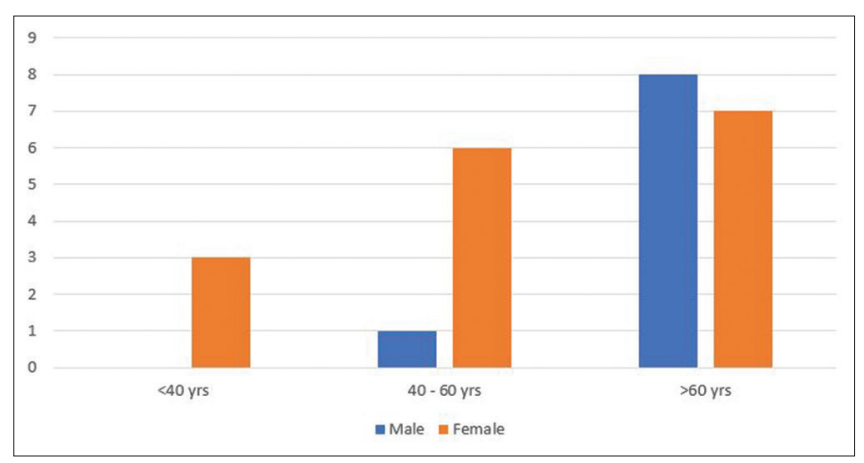

Figure 1: Age and gender distribution of study participants

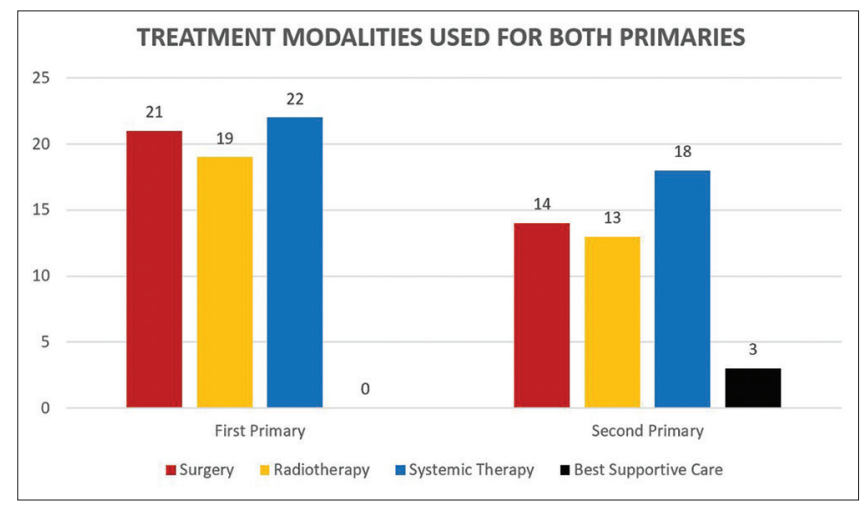

Figure 3: Frequency of use of different treatment modalities in first or second primary

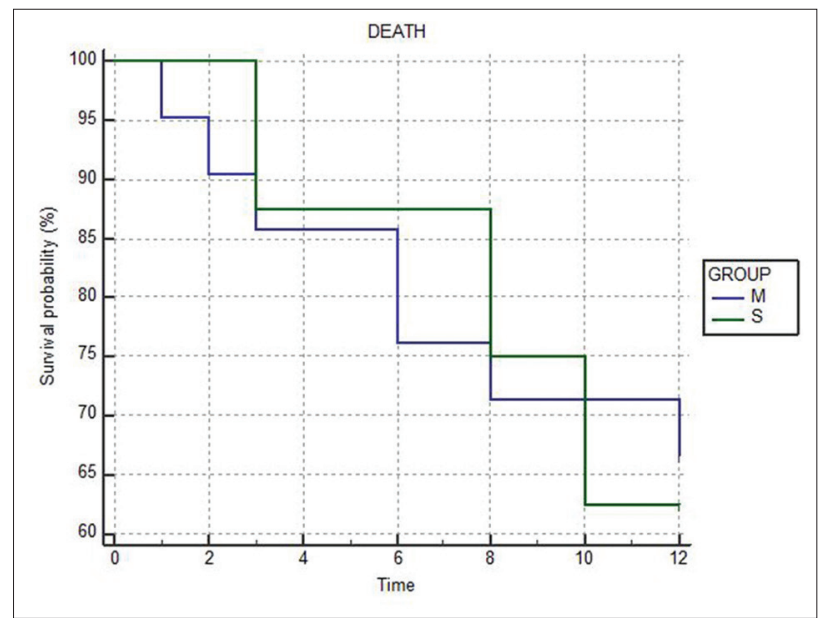

Figure 5: Kaplan-Meier graph comparing survival at 1 year between metachronous (M) and synchronous (S) groups

damage leading to carcinogenesis. The deleterious effects of these treatment modalities as well as of the tumor microenvironment on the patient's immune system may be another important contributing factor allowing future renegade mutant cancer cells from escaping the body's defense mechanisms. Children and young adults may be especially prone to such iatrogenically induced cancers. ${ }^{[17]}$ In our study, a total of 19 patients were treated with radiotherapy for the first primary. Of these, only five developed cancers within the irradiated field; however, the

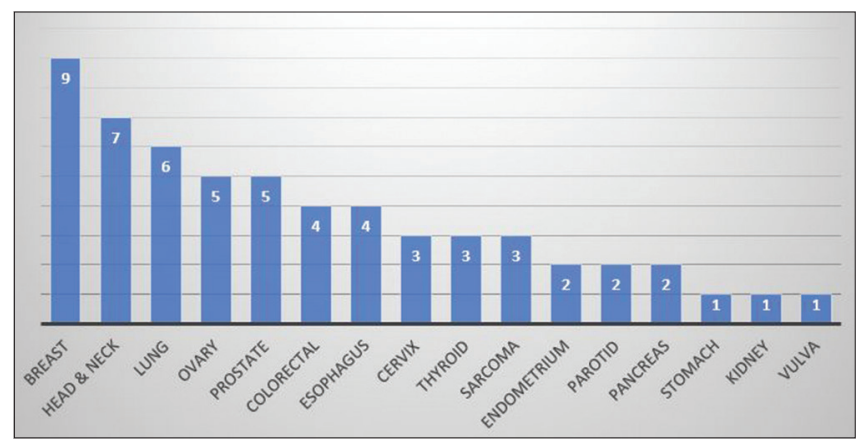

Figure 2: Site-wise distribution of primary cancer sites

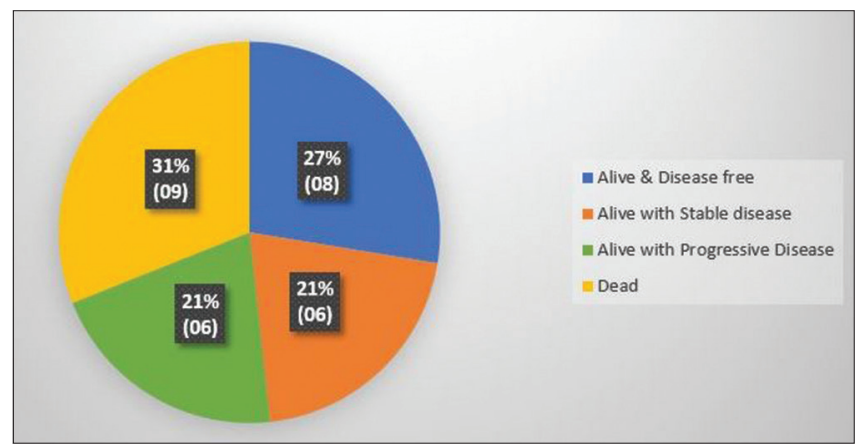

Figure 4: Status of patients at 1 year from diagnosis of second primary

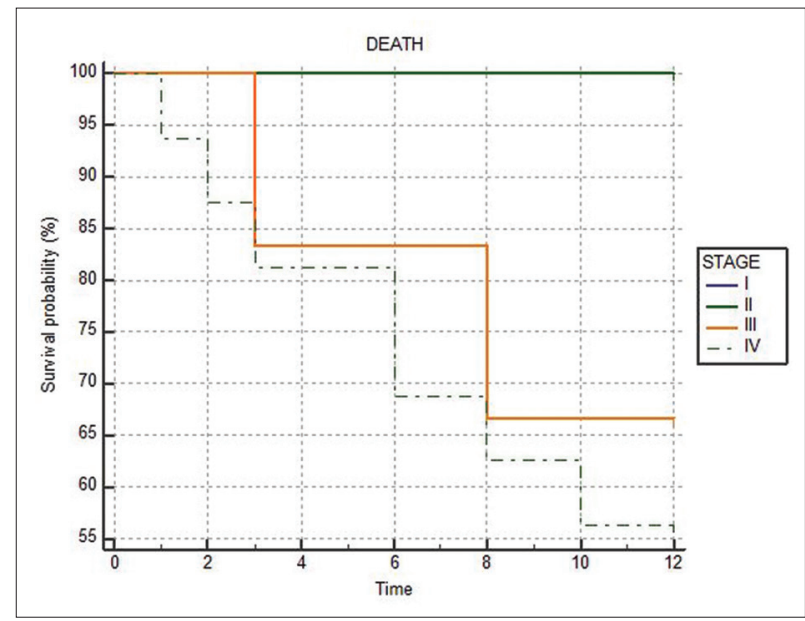

Figure 6: Kaplan-Meier graph comparing survival at 1 year between Stages I to IV of the second primary cancers

time interval of occurrence of these second primaries was too short (5 months to 3 years) to be attributable to their radiation treatment. Radiation-induced solid cancers usually have a latency period of 5-10 years or more. ${ }^{[18]}$ Although the use of tamoxifen in patients of carcinoma breast, especially those above 55 years of age, has been associated with a $2.6 \%$ increased risk of developing endometrial carcinoma, we did not detect any second primary cancers associated with hormone therapy use. ${ }^{[19]}$

To diagnose a second malignancy in the setting of a primary one is difficult and requires good communication between the patient and doctor along with stringent follow-up. Even 
then, the second malignancy can be masked by the symptoms of the first neoplasm and the diagnosis confounded by possibility of local or distant recurrence of first cancer. There are certain clinical pointers that need to be kept in mind when suspecting a second malignancy. Fresh-onset symptoms in patients with exposure to environmental carcinogens (e.g., smoking), suspicion of hematological malignancy after prior chemotherapy (e.g., etoposide, anthracyclines), suspicion of secondary malignancy in patients with prior treatment with ionizing radiation (especially if a new lesion appears in the prior irradiated field), and any new metastatic site of disease after a prolonged state of dormant behavior of the primary malignancy should always be investigated to rule out a second primary cancer. Imaging of asymptomatic patients as a part of follow-up especially with positron emission tomography (PET)-computed tomography (CT) can be helpful in identifying new-onset lesions and give the physician lead time in early diagnosis. However, due to high costs and repeated exposure to radiation, it is difficult to justify its use. Hence, the need for good detailed history and clinical examination can never be overemphasized and the possibility of a new malignancy should always be borne in mind during follow-up of cancer patients. As listed above, use of more stringent surveillance and screening for second cancers as well as modern diagnostic technologies such as PET-CT, image-guided tissue biopsy, and immunohistochemistry have also greatly contributed to increasing the diagnosis of multiple malignancies.

Certain risk factors for second primary in a patient of malignancy that have been identified are younger age at diagnosis of primary cancer, presence of comorbidities, lifestyle, lower stage of first cancer with a long disease-free period, phenomenon of field cancerization, and positive family history. ${ }^{[11]}$

Although a second malignancy can be detected at any age, there is normally a predisposition toward older age as compared to a newly diagnosed first malignancy. Several reports have shown the mean age for reporting second cancer to be around 50 years or above. ${ }^{[20-22]}$ In our study too, the median age at diagnosis of second malignancy was 56 years, with $76 \%$ (22) patients above the age of 50 years. Male predominance has been reported in many data analysis of second malignancies; $;^{[23-25]}$ however, an analysis of the SEER cancer registries ${ }^{[6]}$ from 1971 to 2000 showed the relative risk of developing subsequent cancers to be higher for females than males (1.17 vs. 1.11). In our series, females were the predominant gender with $65.5 \%$ patients with dual cancers being women. This was a direct result of the fact that $31 \%$ (18) of cancers were almost exclusive to the female anatomy (breast, ovary, cervix, and vulva). The high frequency of breast cancer primaries in our study also meant that while females were more common in the age group below 60 , men, whose were mostly affected by head and neck, prostate, and lung cancers, greatly outnumbered the women in the age group above 60 years.
The percentage of tumors in advanced stages (Stage IV) was significantly more in the second primary tumors $(55 \%)$ than the first primary tumors (17\%). An explanation for this could be the delayed diagnosis of the second primary due to its signs and symptoms being mistaken for those of a recurrence of the first primary.

Various series of multiple malignancies have reported varying percentages of synchronous and metachronous cancers. ${ }^{[9,10,26,27]}$ In our study group, metachronous cancers outnumbered synchronous cancers by 2.5 times. There appeared to be no statistical difference in percentage of patients alive or probability of survival at 1 year in the two groups. The more important factor affecting survival at 1 year appears to be the stage of presentation of second cancer, irrespective of whether it is synchronous or metachronous [Figure 5].

The risk of developing a second primary malignancy is varying in different cancer sites and is known to range from $1 \%$ in hepatic cancers to up to $16 \%$ in urinary bladder cancers. ${ }^{[28]}$ Common sites of second primary malignancy after a primary cancer are respiratory, gastrointestinal, and genitourinary malignancies. ${ }^{[19]}$ According to several data analyses, the common primary malignancies seen in a multiple cancer setting are cancers of the breast, prostate, lung, colorectal, and urinary system. ${ }^{[28]}$ In our study too, breast was the most commonly involved site of malignancy. While lung, ovarian, and prostate were also encountered commonly, the most second common site was of head and neck. This is in accordance with cancer statistics of our country where tobacco-related cancers comprise about $30 \%$ of all cancers. ${ }^{[29]}$ Among the 3879 patients seen at our center, those with breast cancer were the most likely to have a second primary cancer with seven patients of dual malignancy in our study having breast as their first primary cancer site. On the other hand, lung cancer was the most likely second primary among all patients of our study with five patients suffering from it as a second primary.

The most common pairs of tumors seen in our literature review were prostate-lung in males and breast-breast or breast-colon in females. ${ }^{[28]}$ Again, in our own series, the breast-colorectal remained the most common cancer pair (3) in females while hypopharynx-lung was most common (2) in males.

Genetic susceptibility is a dominant factor in the etiology of secondary malignancies, and patients with positive family history have an increased genetic susceptibility to develop a second malignancy. There are several syndromes, which group the occurrence of certain cancers together increasing the probability of one preceding the other. Common syndromes include hereditary breast and ovary cancer syndrome (HBOC), Li Fraumeni syndrome, Lynch syndrome, multiple endocrine neoplasia (MEN 1 and MEN 2), and von Hippel-Lindau disease. Each of these syndromes is associated with a specific and characteristic 
genetic abnormality or mutation. Prior treatment for cancer also renders the DNA susceptible to chromosomal rearrangement or loss, leading to chromosomal abnormalities and possible carcinogenesis. ${ }^{[30]}$

Germline and somatic mutations that play an important role in carcinogenesis are also being recognized now as possible targets of treatment. Some of the important mutations with respect to new treatment options and strategies are the use of poly-ADP-ribose polymerase inhibitors in patients whose cancer displays DNA repair defects (BRCA1/2, ATM) or checkpoint inhibitors in tumors with high mutational load as exemplified by microsatellite instability (MSI). MSI is one genetic change that is noticed more frequently in the setting of multiple cancers. ${ }^{[31]}$ Nevertheless, commercial application of this testing to determine risk of multiple malignancy at a high financial cost in the absence of genetic counseling cannot be justified at the moment. ${ }^{[32,33]}$ In our study group, only two patients had a history of cancer in their first- or second-degree relatives. Interestingly, both of these patients had the breast-ovarian cancer combination. While one had a history of her father suffering from lung cancer, the other female not only developed carcinoma breast in her early twenties but she also had an elder sister with a history of breast cancer. It is highly possible that she could be suffering from HBOC. She was offered genetic counseling but did not undergo testing for genetic markers for HBOC.

Continuous exposure of different mucosa to the same risk factor can lead to major dysplastic changes, premalignant and malignant lesions. Tobacco and alcohol are the leading causes of most aerodigestive and urogenital cancers such as head and neck, esophagus, respiratory system, pancreas, urinary bladder, and cervix. ${ }^{[34-36]}$ Field cancerization is a well-established phenomenon where the effect of smoking and alcohol predisposes the entire mucosa of the aerodigestive tract or the transitional cell mucosa of the bladder and lower urinary system to a secondary malignancy. ${ }^{[3]}$ Continuing smoking and alcohol after completing treatment for the primary malignancy increases the risk of second cancer by $35 \% \cdot{ }^{[17,38-40]}$ This is a common phenomenon seen in most lower social class patients and can be attributed to illiteracy and ignorance. In the research of continuous exposure to a known carcinogen, smoking has emerged time and again as a high-ranking culprit. ${ }^{[34-36]}$ Among the patients in our study, 10 had a history of using tobacco, whether smoked or smokeless. Of these, at least six had both such malignancies that could be attributed to tobacco. The occurrence of second primaries in these six cases can probably be explained by field cancerization resultant from tobacco exposure. Alcohol consumption was not recorded.

There are very little data to show survival trends in the patients of multiple malignancies. Survival can also be affected by the advanced age of presentation and other coexisting comorbidities. In our study, no differences in survival probability at 1 year were seen in the metachronous or synchronous groups. Survival in a case of multiple malignancies seems to be related to stage of presentation of each primary and is probably not a function of the presence of multiple cancers itself.

The possible fallacies of our study are that it is retrospective in nature with a sample size not big enough for robust statistical analysis.

\section{Conclusion}

While the incidence of multiple primary cancers appears to be increasing, early diagnosis of a second primary in the background of an existing malignancy remains a challenge. Screening for second malignancies is an attractive option, but the optimal screening modalities with cost-effectiveness in mind elude us for most cancers. ${ }^{[41]}$ With regular monitoring, accompanied by careful history taking, thorough examination, and appropriate investigations, second primary tumors could be detected earlier and with timely intervention might be better managed with improvement in survival. Patient counseling about lifestyle modifications, especially smoking and alcohol cessation, are even more important in cancer survivors than in those without a history of the disease.

Our data can possibly sensitize practicing oncologists toward the prevalence of the dual malignancies and other MPMs in the Indian population and help develop an index of suspicion for their early detection.

\section{Acknowledgments}

Departments of Radiology, Nuclear Medicine and Pathology of Command Hospital (SC), Pune.

\section{Financial support and sponsorship}

The study was funded by Command Hospital (SC), Wanowrie, Pune, Maharashtra, India.

\section{Conflicts of interest}

There are no conflicts of interest.

\section{References}

1. Noh SK, Yoon JY, Ryoo UN, Choi CH, Sung CO, Kim TJ, et al. A case report of quadruple cancer in a single patient including the breast, rectum, ovary, and endometrium. J Gynecol Oncol 2008; 19:265-9.

2. Lee JS, Moon W, Park SJ, Park MI, Kim KJ, Jang LL, et al. Triple synchronous primary cancers of rectum, thyroid, and uterine cervix detected during the workup for hematochezia. Intern Med 2010;49:1745-7.

3. Billroth T. General Surgical Pathology and Therapy in 51 Lectures: A Handbook for Students and Physicians. 14 $4^{\text {th }}$ ed. Berlin, Germany: G. Reimer; 1889. p. 908.

4. Owen LJ. Multiple malignant neoplasms. JAMA 1921;76:1329-33.

5. Warren S. Multiple malignant tumours. A survey of the literature and statistical study. Am J Cancer 1932;16:1358-414. 
6. Moertel CG. Multiple primary malignant neoplasms: Historical perspectives. Cancer 1977;40:1786-92.

7. Moertel CG, Dockerty MB, Baggenstoss AH. Multiple primary malignant neoplasms. I. Introduction and presentation of data. Cancer 1961;14:221-30.

8. Sharma D, Singh G, Kakkar N, Raj S. Second primary malignancy: A retrospective analysis report from a tertiary cancer center of North India. Indian J Cancer 2016;53:595-9.

9. Bagri PK, Singh D, Singhal MK, Singh G, Mathur G, Jakhar SL, et al. Double primary malignancies: A clinical \& pathological analysis report from a regional cancer institute in India. Iran J Cancer Prev 2014;7:66-72.

10. Jena A, Patnayak R, Lakshmi AY, Manilal B, Reddy MK. Multiple primary cancers: An enigma. South Asian J Cancer 2016;5:29-32.

11. Amer MH. Multiple neoplasms, single primaries, and patient survival. Cancer Manag Res 2014;6:119-34.

12. Bajdik CD, Abanto ZU, Spinelli JJ, Brooks-Wilson A, Gallagher RP. Identifying related cancer types based on their incidence among people with multiple cancers. Emerg Themes Epidemiol 2006;3:17.

13. Gaskin HS, Hardy RE, Fletcher RL. Multiple primary malignancies in black patients. J Natl Med Assoc 1981;73:1065-8.

14. Donin N, Filson C, Drakaki A, Tan HJ, Castillo A, Kwan L, et al. Risk of second primary malignancies among cancer survivors in the United States, 1992 through 2008. Cancer 2016;122:3075-86.

15. AIRTUM Working Group. Italian cancer figures, report 2010: Cancer prevalence in Italy. Patients living with cancer, long-term survivors and cured patients. Epidemiol Prev 2010;34:1-88.

16. Hauben EI, Arends J, Vandenbroucke JP, van Asperen CJ, Van Marck E, Hogendoorn PC, et al. Multiple primary malignancies in osteosarcoma patients. Incidence and predictive value of osteosarcoma subtype for cancer syndromes related with osteosarcoma. Eur J Hum Genet 2003;11:611-8.

17. Curtis RE, Freedman DM, Ron E, Ries LA, Hacker DG, Edwards BK, et al., editors. New Malignancies Among Cancer Survivors: SEER Cancer Registries, 1973-2000. Bethesda, MD: National Cancer Institute; 2006. p. 9-14.

18. Hall EJ. Radiobiology for the Radiologist. $5^{\text {th }}$ ed. Philadelphia (PA): Lippincott Williams \& Wilkins; 2000. p. 149.

19. Early Breast Cancer Trialists' Collaborative Group (EBCTCG), Davies C, Godwin J, Gray R, Clarke M, Cutter D, et al. Relevance of breast cancer hormone receptors and other factors to the efficacy of adjuvant tamoxifen: Patient-level meta-analysis of randomised trials. Lancet 2011;378:771-84.

20. Hajdu SI, Hajdu EO. Multiple primary malignant tumors. J Am Geriatr Soc 1968;16:16-26.

21. Berge T, Cederqvist L, Schönebeck J. Multiple primary malignant tumours. An autopsy study of a circumscribed population. Acta Pathol Microbiol Scand 1969;76:171-83.

22. Vyas JJ, Deshpande RK, Sharma S, Desai PB. Multiple primary cancers in Indian population: Metachronous and synchronous lesions. J Surg Oncol 1983;23:239-49.

23. Gursel B, Meydan D, Özbek N, Ozdemir O, Odabas E. Multiple primary malignant neoplasms from the black sea region of turkey. J Int Med Res 2011;39:667-74.

24. Ueno M, Muto T, Oya M, Ota H, Azekura K, Yamaguchi T, et al. Multiple primary cancer: An experience at the cancer institute hospital with special reference to colorectal cancer. Int J
Clin Oncol 2003;8:162-7.

25. Schwartz LH, Ozsahin M, Zhang GN, Touboul E, De Vataire F, Andolenko P, et al. Synchronous and metachronous head and neck carcinomas. Cancer 1994;74:1933-8.

26. Lv M, Zhang X, Shen Y, Wang F, Yang J, Wang B, et al. Clinical analysis and prognosis of synchronous and metachronous multiple primary malignant tumors. Medicine (Baltimore) 2017;96:e6799.

27. Mehdi I, Shah AH, Moona MS, Verma K, Abussa A, Elramih R, et al. Synchronous and metachronous malignant tumours expect the unexpected. J Pak Med Assoc 2010;60:905-9.

28. Hayat MJ, Howlader N, Reichman ME, Edwards BK. Cancer statistics, trends, and multiple primary cancer analyses from the surveillance, epidemiology, and end results (SEER) program. Oncologist 2007;12:20-37.

29. Three Year Report of Population Based and Hospital Based Cancer Registries; 2012-2014. Available from: http://www. ncdirindia.org/. [Last accessed on 2016 May 22].

30. Escobar PA, Smith MT, Vasishta A, Hubbard AE, Zhang L. Leukaemia-specific chromosome damage detected by comet with fluorescence in situ hybridization (comet-FISH). Mutagenesis 2007;22:321-7.

31. Horii A, Han HJ, Shimada M, Yanagisawa A, Kato Y, Ohta H, et al. Frequent replication errors at microsatellite loci in tumors of patients with multiple primary cancers. Cancer Res 1994;54:3373-5.

32. European Academies Science Advisory Council. Direct-To-Consumer Genetic Testing for Health-Related Purposes in the European Union: the View from EASAC and FEAM EASAC policy Report. European Academies Science Advisory Council; 2012. p. 18.

33. Robson ME, Storm CD, Weitzel J, Wollins DS, Offit K; American Society of Clinical Oncology. American Society of Clinical Oncology policy statement update: Genetic and genomic testing for cancer susceptibility. J Clin Oncol 2010;28:893-901.

34. Kelsey JL. Breast and Gynecology Cancer Epidemiology. Boca Raton, FL: CRC Press; 1983.

35. Brinton LA, Blot WJ, Becker JA, Winn DM, Browder JP, Farmer JC Jr., et al. A case-control study of cancers of the nasal cavity and paranasal sinuses. Am J Epidemiol 1984;119:896-906.

36. Office on Smoking and Health. Report of the Surgeon General. DHEW Publication No. (PHS) 79-50066. Washington, DC: U.S. Government, Printing Office; 1979.

37. Slaughter DP, Southwick HW, Smejkal W. Field cancerization in oral stratified squamous epithelium; clinical implications of multicentric origin. Cancer 1953;6:963-8.

38. Liu YY, Chen YM, Yen SH, Tsai CM, Perng RP. Multiple primary malignancies involving lung cancer-clinical characteristics and prognosis. Lung Cancer 2002;35:189-94.

39. Weichert KA, Schumrick D. Multiple malignancies in patients with primary carcinomas of the head and neck. Laryngoscope 1979;89:988-91.

40. Tucker MA, Murray N, Shaw EG, Ettinger DS, Mabry M, Huber $\mathrm{MH}$, et al. Second primary cancers related to smoking and treatment of small-cell lung cancer. Lung cancer working cadre. J Natl Cancer Inst 1997;89:1782-8.

41. Vogel VG. Identifying and screening patients at risk of second cancers. Cancer Epidemiol Biomarkers Prev 2006;15:2027-32. 